\begin{tabular}{|c|l|}
\hline Title & Electric-field-induced flow-aligning state in a nematic liquid crystal \\
\hline Author(s) & Fatriansy ah, Jaka Fajar; Orihara, Hiroshi \\
\hline Citation & $\begin{array}{l}\text { Physical review E, 91(4), 042508 } \\
\text { https://doi.org/L0.1103/PhysRevE.91.042508 }\end{array}$ \\
\hline Issue Date & 2015-05-01 \\
\hline Doc URL & http://hdl.handle.net/2115/59328 \\
\hline Rights & @2015A merican Physical Society \\
\hline Type & article \\
\hline File Information & PhysRevE.91.042508.pdf \\
\hline
\end{tabular}

Instructions for use 


\title{
Electric-field-induced flow-aligning state in a nematic liquid crystal
}

\author{
Jaka Fajar Fatriansyah and Hiroshi Orihara \\ Division of Applied Physics, Faculty of Engineering, Hokkaido University, Sapporo, Hokkaido 060-8628, Japan
}

(Received 18 February 2015; published 30 April 2015)

\begin{abstract}
The response of shear stress to a weak ac electric field as a probe is measured in a nematic liquid crystal under shear flow and dc electric fields. Two states with different responses are clearly observed when the dc electric field is changed at a constant shear rate: the flow aligning and non-flow aligning states. The director lies in the shear plane in the flow aligning state and out of the plane in the non-flow aligning state. Through application of dc electric field, the non-flow aligning state can be changed to the flow aligning state. In the transition from the flow aligning state to the non-flow aligning state, it is found that the response increases and the relaxation time becomes longer. Here, the experimental results in the flow aligning state are discussed on the basis of the Ericksen-Leslie theory.
\end{abstract}

DOI: 10.1103/PhysRevE.91.042508

PACS number(s): $83.80 . \mathrm{Xz}$

\section{INTRODUCTION}

Nematic liquid crystals (NLCs) exhibit many interesting phenomena related to the coupling between the orientations of the elongated molecules and flow. For example, a simple shear flow can align the average orientation, called the director n. In this case, the director is confined in the shear plane and the angle between the director and the flow direction is given by $\theta_{f}=\cos ^{-1}\left(-\gamma_{1} / \gamma_{2}\right) / 2$ [1], where $\gamma_{1}$ is the rotational viscosity and $\gamma_{2}$ is the irrotational viscosity. It is readily seen from this equation that the flow aligning angle $\theta_{f}$ cannot exist for $\left|\gamma_{1} / \gamma_{2}\right|>1$. In this case, the director will tumble or find another stable orientation with the director field being spatially deformed, possibly in accordance with the viscous and elastic coefficients and the boundary condition. Thus, under a simple shear flow, NLCs take either one of two types of states depending on the ratio of $\gamma_{1}$ to $\gamma_{2}$ : the flow aligning state (FAS) and non-flow aligning state (NFAS). Most low-molecular-mass NLCs under shear flow adopt the FAS except for a small number of NLCs such as 7CB and 8CB (4-n-octyl-cyanobiphenyl), though the NFAS is common in liquid crystal polymers [1]. NFAS can be easily transformed to FAS by applying electric fields along the velocity gradient direction in NLCs with positive dielectric anisotropy. The transformation from NFAS to FAS at a critical electric field may be regarded as an electric-field-induced phase transition under shear flow, which is of interest from the viewpoint of nonequilibrium physics.

Basically, the transition is due to competition between the shear flow effect and the electric-field effect, where the latter compensates for the imbalance of the torque produced by the former and finally stabilizes the director orientation back to the shear plane in a strong enough electric field. This transition has been experimentally probed using various methods such as direct observation of flow patterns and disclination density [2-4], rheological measurements [5], and synchrotron x-ray studies [6]. However, it is not easy to clearly distinguish between the FAS and NFAS states by means of the usual rheological measurements. Although the electric-field dependence of shear stress in $8 \mathrm{CB}$ at a constant shear rate, in which an NFAS appears without electric field, was also reported by Negita [7] and Patricio et al. [8]; the change in the shear stress was too small for the transition to be clearly detected. In this study, we measured the shear stress response to an ac electric field as a probe under both shear flow and dc electric fields [9]. These measurements are more sensitive to the change in director orientation and demonstrate that different frequency dispersion curves in the stress response can be clearly observed and the transition from NFAS to FAS can be detected by changing the dc electric field at a constant shear rate. Furthermore, anomalous behaviors near the transition point are reported.

\section{EXPERIMENT}

The nematic liquid crystal $8 \mathrm{CB}$ was purchased from Wako Pure Chemical Industries and used without any further purification. Measurements were carried out by using a parallel plate rotational rheometer (Physica MCR300, Anton Paar). The diameter of the rotating plate and the gap between two parallel plates were 35 and $0.2 \mathrm{~mm}$, respectively. No surface treatment was made. However, we believe that NLC can align due to the shear flow and electric fields except near the surfaces in FAS. Note that in the parallel plate geometry, the shear rate depends on position, and so the shear rate is defined at the edge of the upper plate and the shear stress at the corresponding shear rate is calculated from the mechanical torque by assuming that the sample is Newtonian. All the measurements were made at $37^{\circ} \mathrm{C}$ in the nematic phase. The experimental setup and the geometry are shown in Figs. 1(a) and 1(b), respectively. Electric fields were applied to the sample by using a synthesizer (Model 1940, NF Electric Instruments) and a high-voltage amplifier (4005, NF). In our measurements, dc electric fields were applied to induce the FAS, and a weak ac electric field was also applied to probe the stress response. The total field applied was thus $E_{0}+\Delta E \cos \omega t$. However, migration of ions in the NLC sometimes becomes a problem and reduces the electric field inside the cell. To avoid this, we used a high-frequency ac electric field modulated by the sum of the dc and ac electric fields:

$$
E(t)=\sqrt{2}\left(E_{0}+\Delta E \cos \omega t\right) \cos \omega_{c} t
$$

where $\omega_{c}$ is the angular frequency of the carrier signal. As will be shown in the next section, the induced shear stress is proportional to the square of the applied electric 
(a)

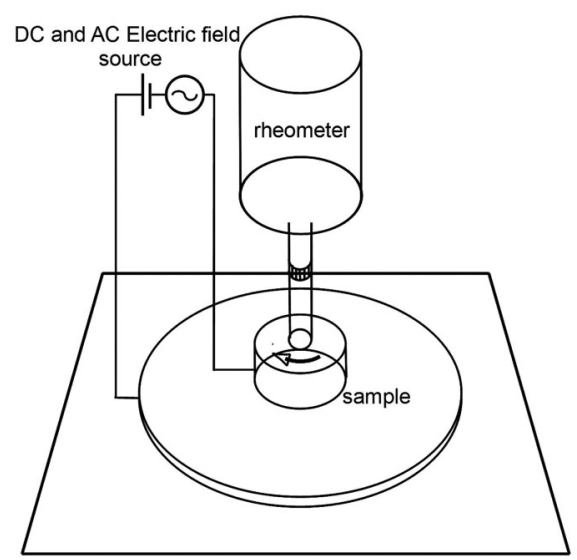

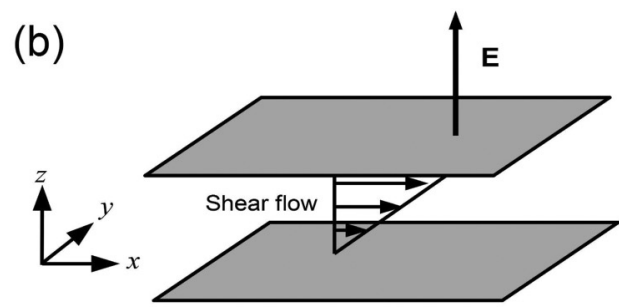

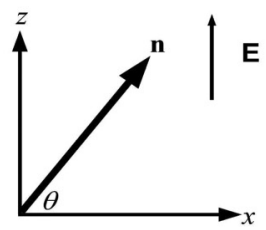

FIG. 1. (a) Experimental setup. The sample is sheared by using a parallel-plate rheometer under dc and ac electric fields. (b) The flow is applied in the $x$ direction, the velocity gradient is in the $z$ direction, and the electric field is applied in the $z$ direction.

field so that the square of $E(t)$ can be approximated by $\left(E_{0}+\Delta E \cos \omega t\right)^{2}$ for $\omega_{c} \gg \omega$. In our measurements, $\omega_{c}$ is chosen to be $6280 \mathrm{rad} \mathrm{s}^{-1}$, which is much higher than the maximum frequency of $\omega=200 \mathrm{rad} \mathrm{s}^{-1}$ in our measurements.

Since $\left(E_{0}+\Delta E \cos \omega t\right)^{2}$ can be expanded to give $E_{0}^{2}+$ $2 E_{0} \Delta E \cos \omega t+\Delta E^{2} / 2+\Delta E^{2} / 2 \cos 2 \omega t$, the corresponding shear stress may be written as

$$
\begin{aligned}
\sigma(t)= & \sigma_{0}+\operatorname{Re}\left[\Delta \sigma_{1,1}(\omega) e^{i \omega t}\right]+\Delta \sigma_{2,0}(\omega) \\
& +\operatorname{Re}\left[\Delta \sigma_{2,2}(\omega) e^{i 2 \omega t}\right]
\end{aligned}
$$

where $\sigma_{0}$ is the shear stress in the absence of the perturbation $\Delta E$, and $\Delta \sigma_{i, j}$ is the stress response of the $i$ th order with respect to $\Delta E$ and the $j$ th harmonic of $\omega$. Therefore, $\Delta \sigma_{1,1}(\omega)$ is proportional to $\Delta E$, and both $\Delta \sigma_{2,0}(\omega)$ and $\Delta \sigma_{2,2}(\omega)$ are proportional to the square of $\Delta E$ for small values of $\Delta E$. The $\omega$ and $2 \omega$ components of the shear stress were obtained by using a vector signal analyzer (HP89410A, Hewlett-Packard).

\section{THEORETICAL RESULTS IN FAS}

In the FAS, the director can be assumed to lie in the shear plane and to be independent of position except near the boundary plates. In this simple case, the shear stress response has been calculated on the basis of the Ericksen-Leslie theory [9]. We briefly summarize the results and note some additional considerations regarding the electric-field-induced FAS in the following. The stress response has not yet been calculated in the NFAS because the director field is complicated.

Under a simple shear flow with an electric field applied in the velocity gradient direction, the angle $\theta$ between the director and the flow direction [see Fig. 1(b)] obeys the following equation [9]:

$$
\gamma_{1} \frac{\partial \theta}{\partial t}=-\frac{1}{2} \dot{\gamma}\left(\gamma_{1}+\gamma_{2} \cos 2 \theta\right)+\frac{1}{2} \varepsilon_{0} \Delta \varepsilon E(t)^{2} \sin 2 \theta,
$$

where $\dot{\gamma}$ is the shear rate, $\varepsilon_{0}$ the dielectric constant of vacuum and $\Delta \varepsilon$ the dielectric anisotropy. From this equation, it is readily seen that the change of $\theta$, which brings about the change of the shear stress, depends on the square of the applied electric field $E(t)$, as described above. The shear stress is calculated from

$$
\begin{aligned}
\sigma_{\alpha \beta}^{(\text {visc })}= & \alpha_{4} A_{\alpha \beta}+\alpha_{1} n_{\alpha} n_{\beta} n_{\mu} n_{\rho} A_{\mu \rho}+\alpha_{5} n_{\alpha} n_{\mu} A_{\mu \beta} \\
& +\alpha_{6} n_{\beta} n_{\mu} A_{\mu \alpha}+\alpha_{2} n_{\alpha} N_{\beta}+\alpha_{3} n_{\beta} N_{\alpha},
\end{aligned}
$$

where $A_{\alpha \beta}=1 / 2\left(\nabla_{\beta} v_{\alpha}+\nabla_{\alpha} v_{\beta}\right)$ and $W_{\alpha \beta}=1 / 2\left(\nabla_{\beta} v_{\alpha}-\right.$ $\nabla_{\alpha} v_{\beta}$ ) are the symmetric and antisymmetric parts of the velocity gradient respectively, $N_{\alpha} \equiv d n_{\alpha} / d t-W_{\alpha \beta} n_{\beta}$ is the rate of change of the director with respect to the background fluid, and $\alpha_{i}(i=1, \ldots, 6)$ are the Leslie viscosity coefficients. In the present case, $\mathbf{n}=(\cos \theta, 0, \sin \theta)$ and $\mathbf{v}=(\dot{\gamma} z, 0,0)$, where the $x$ and $z$ axes are taken along the flow and velocity gradient directions, respectively. Note that the shear stress considered here corresponds to $\sigma_{z x}^{\text {(visc) }}$ in Eq. (4), which is the same as $\sigma(t)$ in Eq. (2).

When $E(t)=E_{0}$, Eq. (3) gives [7]

$\theta_{0}=\tan ^{-1}\left\{\frac{\varepsilon_{0} \Delta \varepsilon E_{0}^{2}+\sqrt{\left(\varepsilon_{0} \Delta \varepsilon E_{0}^{2}\right)^{2}+\dot{\gamma}^{2}\left(\gamma_{2}^{2}-\gamma_{1}^{2}\right)}}{\dot{\gamma}\left(\gamma_{1}-\gamma_{2}\right)}\right\}$.

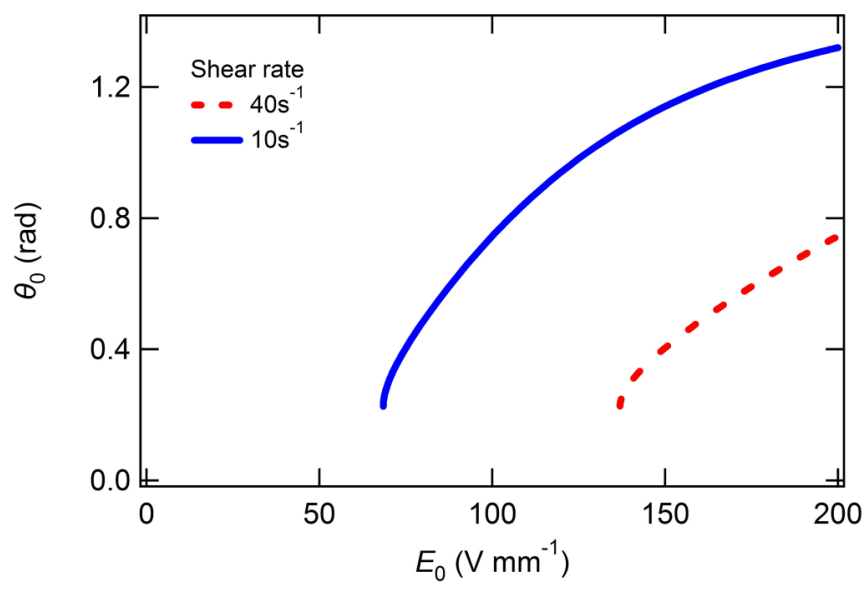

FIG. 2. (Color online) The dc electric-field dependence of the flow alignment angle $\theta_{0}$ in the FAS at shear rates of 10 and $40 \mathrm{~s}^{-1}$ numerically obtained for $8 \mathrm{CB}$ from Eq. (5). 
The calculated dc electric-field dependence of $\theta_{0}$ is shown in Fig. 2. The Leslie coefficients for $8 \mathrm{CB}$ are taken from Ref. [10] $-\alpha_{1}=0.0382$ Pas, $\alpha_{2}=-0.0587$ Pas, $\alpha_{3}=$ $0.0031 \mathrm{Pas}, \alpha_{4}=0.0520 \mathrm{Pas}, \alpha_{5}=0.0472 \mathrm{Pas}$, and $\alpha_{6}=$ $-0.00840 \mathrm{Pas}$ (calculated from the Parodi relation $\alpha_{6}=$ $\left.\alpha_{5}+\alpha_{2}+\alpha_{3}\right)$-and $\Delta \varepsilon=6.5$ is used [11]. In the special case where $E_{0}=0, \theta_{0}$ is reduced to the flow aligning angle $\theta_{f}=$ $\cos ^{-1}\left(-\gamma_{1} / \gamma_{2}\right) / 2$. For 8CB the viscous coefficients are given by $\gamma_{1}=\alpha_{3}-\alpha_{2}=0.0618$ and $\gamma_{2}=\alpha_{6}-\alpha_{5}=-0.0556$, and therefore we have no $\theta_{f}$. However, Eq. (5) has a solution above a critical electric field given by

$$
E_{c}{ }^{2}=\frac{\dot{\gamma} \sqrt{\left(\gamma_{1}^{2}-\gamma_{2}^{2}\right)}}{\varepsilon_{0} \Delta \varepsilon} .
$$

As shown in Fig. 2, $\theta_{0}$ increases with $E_{0}$, and $E_{c}$ increases with $\dot{\gamma}$.

Equation (3) with an ac electric field can be approximately solved by using the perturbation method. Expressing $\theta(t)$ as

$$
\theta(t)=\theta_{0}+\operatorname{Re}\left[\Delta \theta_{1,1} e^{i \omega t}\right]+\Delta \theta_{2,0}+\operatorname{Re}\left[\Delta \theta_{2,2} e^{i 2 \omega t}\right],
$$

we have

$$
\begin{aligned}
\Delta \theta_{1,1}(\omega) & =-\frac{\varepsilon_{0} \Delta \varepsilon E_{0} \Delta E \sin 2 \theta_{0}}{i \omega \tau+1} \frac{\tau}{\gamma_{1}}, \\
\Delta \theta_{2,2}(\omega) & =\frac{(1 / 2)\left|\Delta \theta_{1,1}(\omega)\right|^{2}\left(\dot{\gamma} \gamma_{2} \cos 2 \theta_{0}-\varepsilon_{0} \Delta \varepsilon E_{0} \sin 2 \theta_{0}\right)+\Delta \theta_{1,1} \varepsilon_{0} \Delta \varepsilon E_{0} \Delta E \cos 2 \theta_{0}+\varepsilon_{0} \Delta \varepsilon \Delta E^{2} \sin 2 \theta_{0}}{2 i \omega \tau+1} \frac{\tau}{\gamma_{1}},
\end{aligned}
$$

and

$$
\Delta \theta_{2,0}=\Delta \theta_{2,2}(0),
$$

where the relaxation frequency $1 / \tau$ is defined by

$$
\frac{1}{\tau}=-\frac{1}{\gamma_{1}}\left(\dot{\gamma} \gamma_{2} \sin 2 \theta_{0}+\varepsilon_{0} \Delta \varepsilon E_{0}^{2} \cos 2 \theta_{0}\right) \text {. }
$$

Near the critical electric field, the relaxation frequency is proportional to $\sqrt{E_{0}-E_{c}}$, which is obtained from Eqs. (5), (6), and (9). It should be emphasized that the relaxation frequency becomes zero at the transition point, but the behavior is singular as a function of $E_{0}$. By using the above equations together with Eq. (4), each shear stress in Eq. (2) is written as follows:

$$
\begin{aligned}
\sigma_{0}= & \dot{\gamma}\left[\alpha_{1} \sin ^{2} \theta_{0} \cos ^{2} \theta_{0}+\frac{1}{2}\left\{\alpha_{4}+\left(\alpha_{5}-\alpha_{2}\right) \sin ^{2} \theta_{0}\right.\right. \\
& \left.\left.+\left(\alpha_{3}+\alpha_{6}\right) \cos ^{2} \theta_{0}\right\}\right] \sin 2 \theta_{0}, \\
\Delta \sigma_{1,1}(\omega)= & \dot{\gamma}\left[\alpha_{1} \cos 2 \theta_{0}-\frac{1}{2}\left(\alpha_{2}+\alpha_{3}\right)\right. \\
& \left.+i \omega\left(\alpha_{3} \cos ^{2} \theta_{0}-\alpha_{2} \sin ^{2} \theta_{0}\right)\right] \Delta \theta_{1,1}(\omega), \\
\Delta \sigma_{2,2}(\omega)= & \frac{\dot{\gamma}}{2}\left[\alpha_{1} \sin 4 \theta_{0}-2\left(\alpha_{2}+\alpha_{3}\right) \sin 2 \theta_{0}+2 i \omega\left(\alpha_{3} \cos ^{2} \theta_{0}\right.\right. \\
& \left.\left.-\alpha_{2} \sin ^{2} \theta_{0}\right)\right] \Delta \theta_{2,2}(\omega)+\frac{\dot{\gamma}}{2}\left[\alpha_{1} \cos 4 \theta_{0}-\left(\alpha_{2}+\alpha_{3}\right)\right. \\
& \left.\times \cos 2 \theta_{0}-i \omega\left(\alpha_{2}+\alpha_{3}\right) \sin 2 \theta_{0}\right]\left|\Delta \theta_{1,1}(\omega)\right|^{2},
\end{aligned}
$$

$$
\Delta \sigma_{2,0}=\Delta \sigma_{2,2}(0)
$$

To compare the above theoretical results with experimental ones, we need to consider the parallel-plate geometry of the rheometer used in our experiment. For this geometry, the apparent shear stress is given by [12]

$$
\Delta \sigma_{i, j}^{(R)}(\omega)=\frac{4}{\dot{\gamma}_{R}^{3}} \int_{0}^{\dot{\gamma}_{R}} \Delta \sigma_{i, j}(\omega, \dot{\gamma}) \dot{\gamma}^{2} d \dot{\gamma},
$$

where $\dot{\gamma}_{R}$ is the shear rate at the edge of the rotating disk. The numerically calculated results are presented in the next section, where the superscript " $(R)$ " on the left-hand side of Eq. (11) will be omitted for simplicity.

\section{EXPERIMENTAL RESULTS AND DISCUSSION}

Let us first check the linear relationships between $\Delta E$ and $\Delta \sigma_{1,1}$, and between $\Delta E^{2}$ and $\Delta \sigma_{2,2}$. Figures $3(\mathrm{a})$ and $3(\mathrm{~b})$ show $\Delta E$ vs $\left|\Delta \sigma_{1,1}\right|$ and $\Delta E^{2}$ vs $\left|\Delta \sigma_{2,2}\right|$, respectively, at
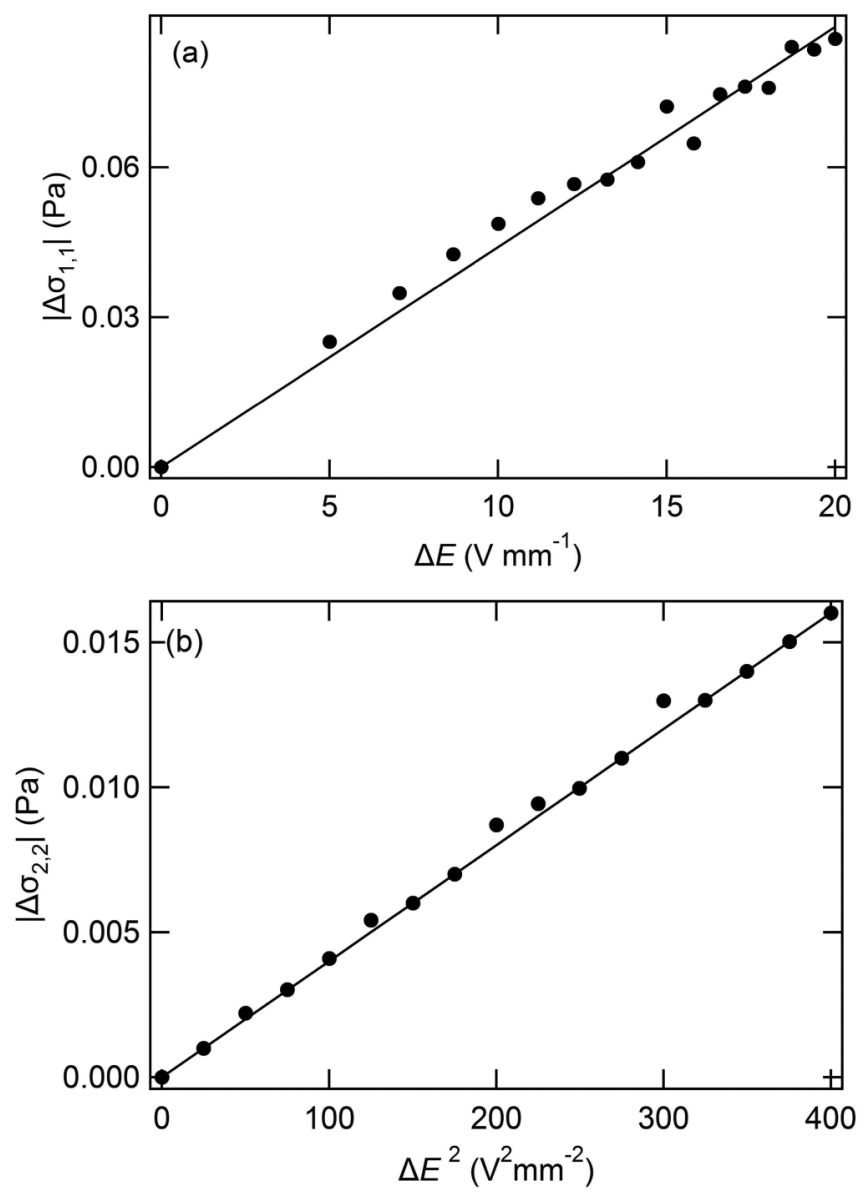

FIG. 3. (a) Dependence of $\left|\Delta \sigma_{1,1}\right|$ on $\Delta E$ at $E_{0}=100 \mathrm{~V} \mathrm{~mm}^{-1}$ and (b) $\left|\Delta \sigma_{2,2}\right|$ on $\Delta E^{2}$ at $E_{0}=0 \mathrm{~V} \mathrm{~mm}^{-1}$ at a shear rate of $10 \mathrm{~s}^{-1}$ at a frequency of $\omega=0.63 \mathrm{rad} \mathrm{s}^{-1}$. Linear relationships are obtained at low electric fields. 

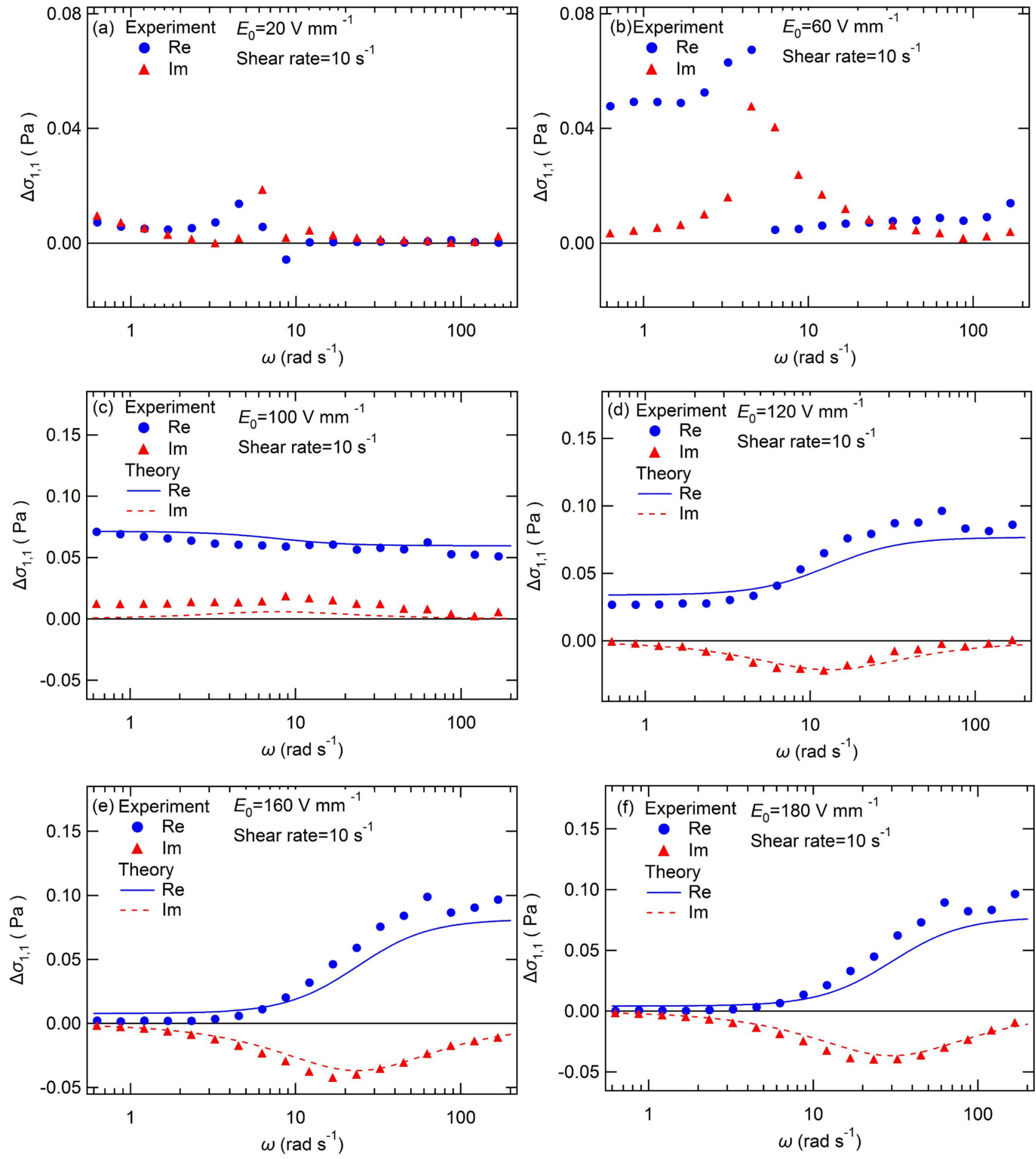

FIG. 4. (Color online) Frequency dispersions of the first-order harmonic response $\Delta \sigma_{1,1}(\omega)$ at a shear rate of $10 \mathrm{~s}^{-1}$ for several dc electric fields. The dispersions are clearly distinguishable between NFAS $(a, b)$ and FAS (c-f). Solid lines for the FAS are calculated on the basis of the Ericksen-Leslie theory.

$\dot{\gamma}=10 \mathrm{~s}^{-1}, E_{0}=100 \mathrm{~V} \mathrm{~mm}^{-1}$, and $\omega=0.63 \mathrm{rad} \mathrm{s}^{-1}$. The linearities are good and the amplitude of the ac electric field for all the measurements is determined to be $\Delta E=14.1 \mathrm{~V} \mathrm{~mm}^{-1}$. The dc electric-field dependence of the frequency dispersion of $\Delta \sigma_{1,1}(\omega)$ at the shear rate of $10 \mathrm{~s}^{-1}$ is shown in Fig. 4. The critical electric field is $E_{c}=68 \mathrm{~V} \mathrm{~mm}^{-1}$ as calculated from Eq. (6). It can be seen that the frequency dispersions below and above the critical electric field are quite different. In the NFAS below this field [Figs. 4(a) and 4(b)], an anomalous change is observed at around $5 \mathrm{rad} \mathrm{s}^{-1}$ in both the real and imaginary parts. However, in the FAS above the critical field [Figs. 5(c)-5(f)], the change is smooth. The behavior of frequency dispersion in the FAS is almost the same as that measured in 5CB (4-n-pentyl-cyanobiphenyl), which adopts 


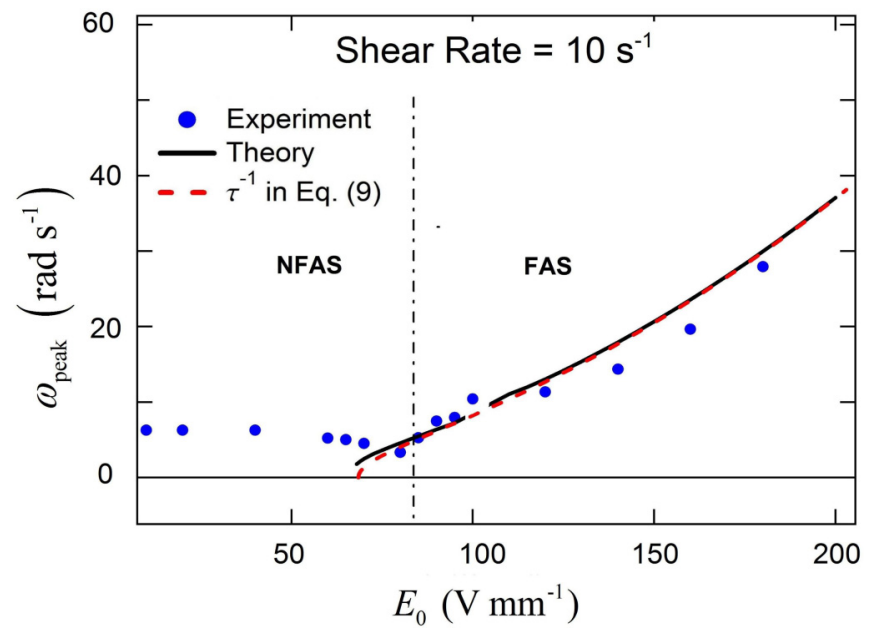

FIG. 5. (Color online) $E_{0}$ dependence of the peak or dip frequency of the imaginary part, $\omega_{\text {peak }}$, where dots are experimental results, a solid line is obtained from calculated frequency dispersion curves using Eq. (11), and a dashed line is $1 / \tau$ calculated from Eq. (9).

the FAS even in the absence of a dc electric field [9]. In $5 \mathrm{CB}$ we have already observed a characteristic behavior under shear flow and dc electric fields: The real part of the frequency dispersion has a plateau at high frequencies. The appearance of the plateau is understood from Eqs. (8a) and (10b) as follows. $\Delta \theta_{1,1}(\omega)$ given by Eq. (8a) is the Debye type which goes to zero at high frequencies, but $\Delta \sigma_{1,1}(\omega)$ differs from it because in Eq. (10b) there is an $i \omega$ term multiplied by $\Delta \theta_{1,1}(\omega)$, which gives a nonzero constant at the limit of $\omega \rightarrow \infty$. The origin of the $i \omega$ term is the time derivative terms in $\alpha_{2} n_{z} N_{x}+\alpha_{3} n_{x} N_{z}=$ $\alpha_{2} n_{z}\left(d n_{x} / d t-n_{z} / 2\right)+\alpha_{3} n_{x}\left(d n_{z} / d t-n_{x} / 2\right)$ of Eq. (4) with $\alpha=z$ and $\beta=x$ in our case. This equation also tells us why the plateau is not observed in the NFAS. In the NFAS the director is thought to be out of the shear plane to some degree, indicating that $n_{x}$ and/or $n_{z}$ should be small. The theoretical curves for the FAS calculated from Eqs. (8a), (10b), and (11) are shown, and are in good agreement with the experimental results.

Figure 5 shows the $E_{0}$ dependence of the peak or dip frequency of the imaginary part, $\omega_{\text {peak }}$, where dots represent the experimental results, the solid line is obtained from the above calculated frequency dispersion curves using Eq. (11), and the dashed line is $1 / \tau$ calculated from Eq. (9). A slowing down of the relaxation time is observed in the FAS, as is expected from Eqs. (5) and (9). At around $100 \mathrm{~V} \mathrm{~mm}^{-1}$ the solid line disappears because the peak changes to the dip in the imaginary part there, and so it is difficult to obtain the peak or dip position. At the critical point, the value of the dashed line becomes zero with the singularity of $\sqrt{E_{0}-E_{c}}$, but that of the solid line is finite because it is averaged out by the integration of Eq. (11). On the other hand, in the experimental results the transition point $\left(82 \mathrm{~V} \mathrm{~mm}^{-1}\right)$ between NFAS and FAS seems to be located above the calculated critical point $\left(68 \mathrm{~V} \mathrm{~mm}^{-1}\right)$. The reasons for this may be as follows: The FAS changes to the NFAS before reaching the calculated critical point due to the boundary condition, and/or the parameters used for the numerical calculations are not suitable for our sample. Except for the details, good agreement is obtained between

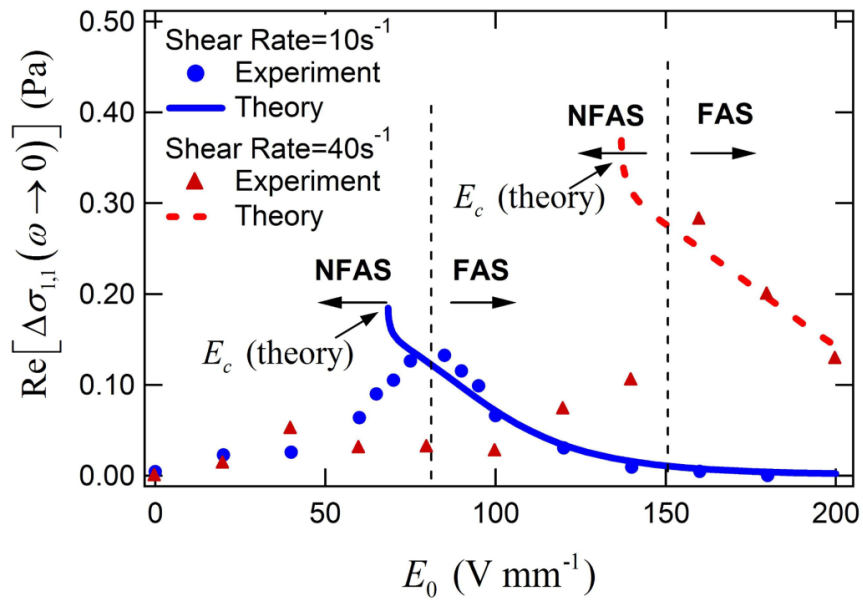

FIG. 6. (Color online) $E_{0}$ dependencies of the first-order response at very low frequency $\left(0.63 \mathrm{rad} \mathrm{s}^{-1}\right.$ for the experiment and exactly zero for the theory), $\operatorname{Re}\left[\Delta \sigma_{1,1}(\omega \rightarrow 0)\right]$, for shear rates of 10 and $40 \mathrm{~s}^{-1}$.

experiment and theory in the FAS, while in the NFAS, the relaxation frequency is relatively low and almost constant.

Next, let us examine in detail the $E_{0}$ dependence of the real part of $\Delta \sigma_{1,1}(\omega)$ in the low-frequency region. The experimentally obtained real part at $0.63 \mathrm{rad} \mathrm{s}^{-1}$ and the theoretically calculated one at zero frequency are shown in Fig. 6 for the shear rates of 10 and $40 \mathrm{~s}^{-1}$. In the FAS the response at low frequencies increases as the transition point is approached and peaks around the transition point for both of the two shear rates. The increase near the transition point in the FAS is reproduced by the theory. On the other hand, the increase in the NFAS may also be due to a pretransitional effect, which is beyond the present theory. The transition electric field at the shear rate of $40 \mathrm{~s}^{-1}$ is higher than that at $10 \mathrm{~s}^{-1}$, as is expected from Eq. (6).

Figure 7 shows the frequency dispersion of the second-order response $\Delta \sigma_{2,2}(\omega)$. In the NFAS [Figs. 7 (a) and $7(\mathrm{~b})$ ] an anomalous change is seen around $3 \mathrm{rad} \mathrm{s}^{-1}$ as well as in the first-order response. In the FAS [Figs. 7(c)-7(f))], the experimental behavior is almost reproduced by the theory. In the second-order response, however, the frequency dispersion becomes more complicated at high frequencies where the real and imaginary parts take a maximum [see Fig. 7(c)], as Eq. (10c) contains $\left|\Delta \theta_{1,1}(\omega)\right|^{2}$. There is a plateau in the second-order response as well as in the first-order response. It should be noted that the plateau is due to the term $i \omega$ multiplied by $\Delta \sigma_{2,2}(\omega)$ in Eq. (10c), but not to the one multiplied by $\left|\Delta \theta_{1,1}(\omega)\right|^{2}$ because $\left|\Delta \theta_{1,1}(\omega)\right|^{2} \propto \omega^{-2}$ at high frequencies. In Fig. 8, the $E_{0}$ dependence of the real part of $\Delta \sigma_{2,2}(\omega)$ in the low-frequency region is shown, where the experimental data are obtained at $0.3 \mathrm{rad} \mathrm{s}^{-1}$ and the theoretical curves are calculated at zero frequency for the shear rates of 10 and $40 \mathrm{~s}^{-1}$. In the FAS, the second-order response is negative, unlike the first-order response, for both the shear rates of 10 and $40 \mathrm{~s}^{-1}$, but the details are different: At $10 \mathrm{~s}^{-1}$ the absolute value of the response increases near the transition point, which may be related to the slowing down, whereas at $40 \mathrm{~s}^{-1}$ it is almost constant as the measurement is taken only near the transition point. 

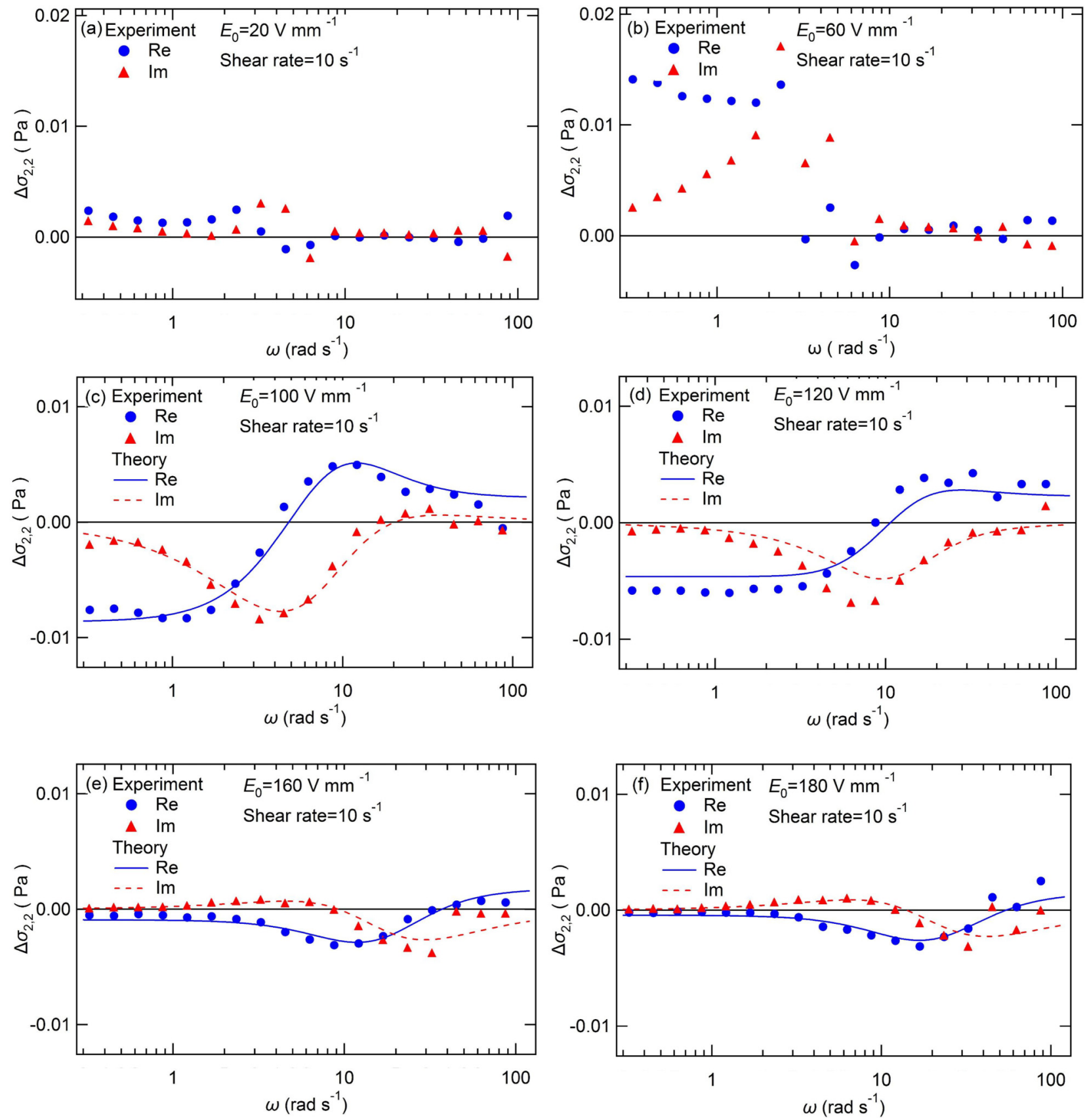

FIG. 7. (Color online) Frequency dispersions of the second-order harmonic response $\Delta \sigma_{2,2}(\omega)$ at a shear rate of $10 \mathrm{~s}^{-1}$ for several dc electric fields. The dispersions are clearly distinguishable between the NFAS (a,b) and the FAS (c-f). Solid lines in the FAS are calculated on the basis of the Ericksen-Leslie theory.

Finally, we show the shear stress without ac electric fields $\sigma_{0}$ in Eq. (2) as a function of $E_{0}$ in Fig. 9, from which it is difficult to distinguish between FAS and NFAS. This clearly shows that the stress response is sensitive to the change in the director field. It is worthwhile to point out that Fig. 9 is closely related to Figs. 6 and 8. At the limit of $\omega \rightarrow 0$, Eq. (2) becomes

$$
\sigma\left(E_{0}+\Delta E\right)=\sigma\left(E_{0}\right)+\Delta \sigma_{1,1}(0)+2 \Delta \sigma_{2,2}(0),
$$

where we have used Eq. (10d) and note that $\sigma_{0}=\sigma\left(E_{0}\right)$. Taking into account that $\Delta \sigma_{1,1} \propto \Delta E$ and $\Delta \sigma_{2,2} \propto \Delta E^{2}$, we have $\Delta \sigma_{1,1}(0)=\sigma^{\prime}\left(E_{0}\right) \Delta E$ and $\Delta \sigma_{2,2}(0)=\sigma^{\prime \prime}\left(E_{0}\right) \Delta E^{2} / 4$. From the latter equation it is easily understood that $\Delta \sigma_{2,2}(0)$ should be negative because $\sigma_{0}=\sigma\left(E_{0}\right)$ is a convex function in the FAS, as is seen from Fig. 9.

\section{CONCLUSIONS}

We have investigated the field-induced transition from the NFAS to FAS by measuring the shear stress response to an ac electric field. Totally different frequency dispersions in the two states were observed for both the first- and secondorder responses. The slowing down of relaxation time and the increased zero-frequency response were found near the transition point as well as in equilibrium phase transitions. 


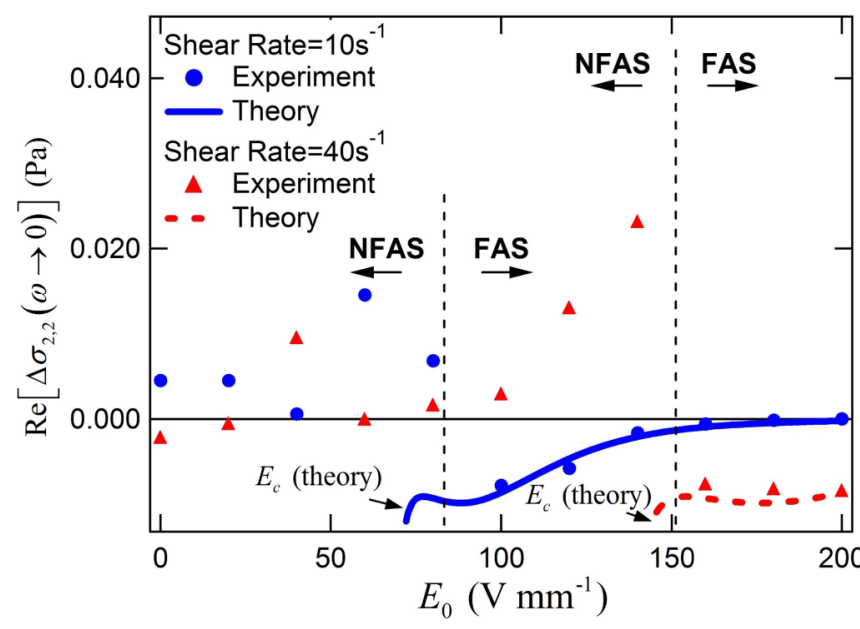

FIG. 8. (Color online) $E_{0}$ dependencies of the second-order response at a very low frequency $\left(0.3 \mathrm{rad} \mathrm{s}^{-1}\right.$ for the experiment and exactly zero for the theory), $\operatorname{Re}\left[\Delta \sigma_{2,2}(\omega \rightarrow 0)\right]$, for shear rates of 10 and $40 \mathrm{~s}^{-1}$.

The experimental results in the FAS were in good agreement with the calculated results based on the Ericksen-Leslie theory, including the plateau observed at high frequencies. As for NFAS, it is necessary to elucidate the director field to calculate the stress response. Our measurement method is expected to be useful for studying nonequilibrium phase transitions under shear flow.

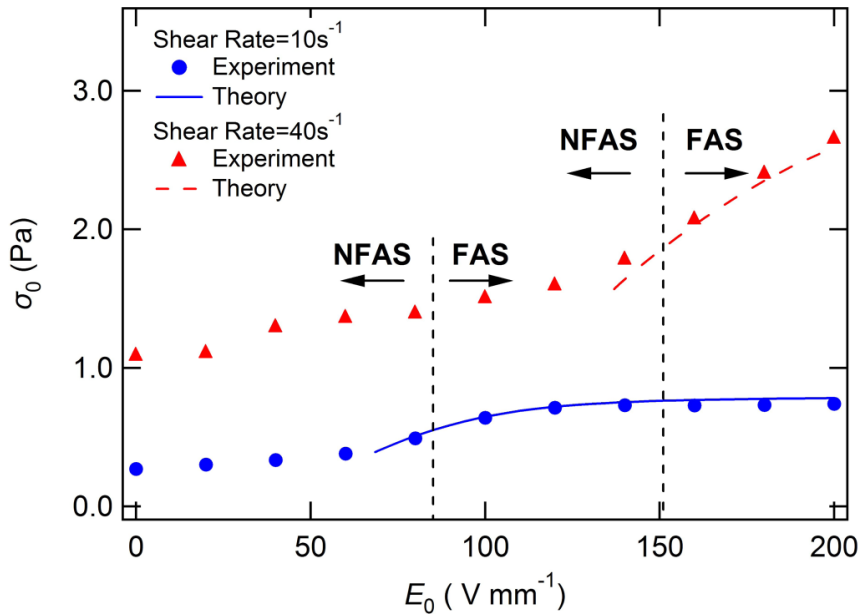

FIG. 9. (Color online) $E_{0}$ dependencies of $\sigma_{0}$ at shear rates of 10 and $40 \mathrm{~s}^{-1}$. It is difficult to distinguish the FAS and NFAS based on $\sigma_{0}$

\section{ACKNOWLEDGMENTS}

This work was supported by JSPS KAKENHI Grants No. 25103006 and No. 26289032. J. F. Fatriansyah would like to thank the Japan International Cooperation Agency (JICA) for the support.
[1] I. K. Yang and A. D. Shine, Macromolecules 26, 1529 (1993).

[2] P. T. Mather, D. S. Pearson, and R. G. Larson, Liq. Cryst. 20, 527 (1996).

[3] P. T. Mather, D. S. Pearson, and R. G. Larson, Liq. Cryst. 20, 539 (1996).

[4] K. Skarp, T. Carlsson, S. T. Lagerwall, and B. Stebler, Mol. Cryst. Liq. Cryst. 66, 199 (1981).

[5] D. J Ternet, R. G. Larson, and L. G. Leal, Rheol. Acta 38, 183 (1999).
[6] C. R. Safinya, E. B. Sirota, R. Plano, and R. F. Bruinsma, J. Phys.: Condens. Matter 2, SA365 (1990).

[7] K. Negita, Mol. Cryst. Liq. Cryst. 300, 163 (1997).

[8] P. Patricio, Liq. Cryst. 39, 25 (2012).

[9] J. F. Fatriansyah, Y. Sasaki, and H. Orihara, Phys. Rev. E 90, 032504 (2014).

[10] J. F. Fatriansyah and H. Orihara, Phys. Rev. E 88, 012510 (2013).

[11] B. R. Ratna and R. Shashidar, Pramana 6, 278 (1975).

[12] H. Orihara, F. Yang, Y. Takigami, Y. Takikawa, and Y. H. Na, Phys. Rev. E 86, 041701 (2012). 